\title{
SCIENTIFIC REPORTS

\section{Genome-wide unique insertion sequences among five Brucella species and demonstration of differential identification of Brucella by multiplex PCR assay}

Soumya Paul ${ }^{1}$, Bhavani Venkataswamachari Peddayelachagiri ${ }^{1}$, Madhurjya Gogoi ${ }^{2}$, Sowmya Nagaraj ${ }^{1}$, Shylaja Ramlal ${ }^{1}$, Balakrishna Konduru ${ }^{1}$ \& Harsh V. Batra ${ }^{1 *}$

Brucellosis is a neglected zoonotic disease caused by alpha proteobacterial genus Brucella comprising of facultative intracellular pathogenic species that can infect both animals and humans. In this study, we aimed to identify genome-wide unique insertion sequence (IS) elements among Brucella abortus, B. melitensis, B. ovis, B. suis and B. canis for use in species differentiation by conducting an intensive in silico-based comparative genomic analysis. As a result, 25, 27, 37, 86 and 3 unique ISs were identified respectively and they had a striking pattern of distribution among them. To explain, a particular IS would be present in four species with $100 \%$ identity whereas completely absent in the fifth species. However, flanking regions of that IS element would be highly identical and conserved in all five species. Species-specific primers designed on these flanking conserved regions resulted in two different amplicons grouping the species into two: one that possesses IS and the other that lacks it. Seeking for species-specific amplicon size for particular species was sufficient to identify it irrespective of biovar. A multiplex PCR developed using these primers resulted in successful differentiation of the five species irrespective of biovars with significant specificity and sensitivity when examined on clinical samples.

Brucella, the etiological agent of a classical zoonotic disease named brucellosis, is an alpha-2 proteobacteria. The genus Brucella includes twelve species: Brucella abortus, B. melitensis, B. suis, B. canis, B. ovis, B. neotomae, B. microti, B. pinnipedialis, B. ceti, B. inopinata, B. papionis, and B. vulpis ${ }^{1}$. These species are aerobic, facultative, intracellular pathogens with specific host preferences for infection and are classified based on diversity in terms of host specificity, phenotypic traits, and pathogenicity. Of these species, B. abortus, B. melitensis and B. suis are further classified into eight $(1-7,9)$, three (1-3) and five (1-5) biovars, respectively. They exhibit a high degree (99\%) of genetic homogeneity, as demonstrated by comparative whole genome analysis ${ }^{2}$.

Although genetic polymorphisms in Brucella are very limited, they have been satisfactorily used in discriminating and genotyping Brucella species ${ }^{3-9}$. Insertion sequences (ISs) are one such genetic element that are reasonably useful in discriminating Brucella species with few limitations. Although the role of IS elements and the reason for their occurrence are highly debated, their distribution has impacted genome evolution.

IS711 is a characteristic IS element among Brucella species. The variable copy numbers and positions of IS elements among species have been reported to render species-associated polymorphisms that have shown promising application in Brucella molecular typing and identification. B. melitensis and B. suis contain seven complete IS711 copies $^{10}$. B. abortus carries six complete copies and one truncated IS711 copy ${ }^{11}$, B. ceti and B. pinnipedialis carry more than 20 copies $^{12,13}$ and B. ovis carries 38 copies $^{14}$. In the 1990s, IS711 was shown to be highly stable, and only under experimental conditions was this IS element transposed in B. ovis and B. pinnipedialis but not in $B$. melitensis or B. abortus ${ }^{10}$. IS711 is characteristic of $B$. abortus and is present in variable numbers and positions but is always present within a given species ${ }^{15}$. The stability of IS711 in the genome was exploited in the development of the famous AMOS multiplex PCR assay to differentiate B. abortus, B. melitensis, B. ovis and B. suis from each 
other $^{16}$. Thus, the stability of IS711 was not only relevant for Brucella typification: its mobility was implicated in the generation of genetic diversity and speciation, as shown by the distribution of IS711 among Brucella species. However, in 2011, Mancilla and coworkers reported the mobility of IS711 in B. abortus field strains under natural conditions, causing polymorphism within the strains of B. abortus. The study reported B. abortus strains isolated from cattle carrying seven copies of complete IS711 instead of six copies, and this new copy was found to be located in a completely new locus that was not previously reported to harbor IS711. The reason for the presence of this one additional copy of IS was predicted to be replicative transposition within the genome ${ }^{15}$. However, in another study, novel insertion of IS711 within the Omp31 gene of an atypical B. ovis isolate recovered from an infected ram in Hungary was encountered and was not correctly identified in the Bruce-ladder multiplex PCR assay ${ }^{17}$. Based on these new case reports, the fact that the most acclaimed IS element, IS711, could be a transposon element in Brucella species was established, and its reliability in differentiating Brucella species was weakened as it demonstrated polymorphism within the strains of a species. With this, the diagnostic application of IS711 reveals the need for a more improved approach to decipher polymorphisms among Brucella species that is even more unique and reliable in differentiating species, even at the biovar level, if possible.

In the current study, we describe a novel in silico-based comparative genomic analysis approach to decipher species-associated unique and precise DNA block distributions among five species of Brucella, namely, B. abortus, B. melitensis, B. ovis, B. suis and B. canis irrespective of biovars. B. abortus, B. melitensis, B. suis and B. canis were selected as representative zoonotic species of Brucella, and B. ovis was selected as a representative species of non-zoonotic Brucella. Due to the unavailability of the remaining seven species in our repository, they were not included in this study. Preliminary in silico characterization of the conserved flanking regions indicated that the DNA blocks were inserted in transposition sites, indicating that the DNA blocks were actually IS elements. This was achieved by conducting an intensive in silico-based comparative genomic analysis of Brucella species using the Perl language and the vast data available online on whole genome sequences of Brucella species. The distribution of the precise stretch of DNA blocks among the five species was unique, as a stretch of nucleotide sequence was embedded within a conserved region of the genome in four of five species but absent in the fifth species. However, the conserved flanking regions (where the primer pairs are located) demonstrated 100\% homology in all five species. Furthermore, these highly specific nucleotide sequences were evaluated for their ability to detect targets, and a novel multiplex PCR was developed wherein the five species were differentiated irrespective of biovars.

\section{Materials and Methods}

Bacterial strains. The Brucella strains (Table 1) were clinical isolates preserved in the repository of the Defence Food Research Laboratory (DFRL). The clinical strains recovered from infected animals and patients were identified by biochemical tests and DNA sequencing. Reference strains of Pseudomonas aeruginosa, P. azotogenesis, Aeromonas hydrophila, Proteus mirabilis, Citrobacter freundii and Yersinia enterocolitica were used as negative controls in the specificity study. All bacterial strains were aseptically recovered from glycerol stocks and grown in trypticase soy broth (TSB) overnight at $37^{\circ} \mathrm{C}$. Genomic DNA from harvested cultures was prepared using a QIAamp DNA Mini Kit (Qiagen, Germany) according to the manufacturer's instructions. DNA samples were determined using a Nanodrop ${ }^{\mathrm{TM}} 1000$ spectrophotometer (Thermo Scientific, MA, USA), stored at $-20^{\circ} \mathrm{C}$ and used in PCR.

Biosafety procedures. All procedures using Brucella strains were performed in a biosafety level 3 laboratory. Procedures for culture of non-Brucella species and DNA extraction were conducted in a class II type A2 biological cabinet (Thermo Scientific, USA).

Genomic analysis of Brucella species. Whole genome sequences (WGSs) of B. abortus A13334, B. melitensis ATCC 23457, B. ovis ATCC 25840, B. suis 1330, B. suis ATCC 23445, B. suis VBI22 and B. canis ATCC 23365 were used for in silico mining of Brucella species-specific targets (Supplementary Table 1). Briefly, the whole genome sequences of the selected Brucella strains were cut into fragments of 1000 bp by implementation of a script written in the Perl programming language. The nucleotide fragments of each species (for instance, $B$. abortus) were aligned against the genomic sequences of all other biovars within the species using the BLASTN program. Nucleotide fragments that matched all biovars with an E-value less than $10^{-200}$ were identified as highly specific targets for $B$. abortus irrespective of biovars (Fig. 1). The procedure was repeated with WGSs of $B$. melitensis, B. suis, B. canis and B. ovis. Thus, the conserved DNA fragments from each species of Brucella were determined and further aligned against the genomic sequences of the other four species to evaluate their specificity.

Analysis of Brucella species-specific DNA fragments. The determined species-specific fragments of Brucella were analyzed for conservation and variation by multiple sequence alignment using the CLUSTALW tool available on the EMBL-EBI website (http://www.ebi.ac.uk/Tools/msa/clustalw2/) and by BLAST analysis against the vast WGS database available in the National Center for Biotechnology Information database (http:// www.ncbi.nlm.nih.gov/) and the Broad Institute (http://www.broadinstitute.org). The respective protein(s) or peptide(s) encoded by each of the species-specific fragments of Brucella were determined using Gene Runner 3.0 software (http://www.generunner.net/). Thus, the determined protein(s) or peptide(s) sequences were analyzed by BLASTP analysis of the National Center for Biotechnology Information (http://www.ncbi.nlm.nih.gov/). Protein sequence features, including the identification of conserved domains, protein superfamily and further protein classification, were performed using the National Center for Biotechnology Information.

Primer design. Primers for specific PCR amplification of Brucella species-specific fragments were designed using Gene Runner 3.0 software (http://www.generunner.net/). The primers were designed to have $\mathrm{T}_{\mathrm{m}}$ values ranging from $52-68^{\circ} \mathrm{C}$ and $\Delta \mathrm{G}$ values for primer duplex below $-10 \mathrm{kcal} / \mathrm{mol}$ and not to form hairpin loops or 


\begin{tabular}{|c|c|c|c|}
\hline Reference Strains & Provider & $\begin{array}{l}\text { No. of strains } \\
\text { (n) }\end{array}$ & $\begin{array}{l}\text { PCR product size (bp) with } \\
\text { species-specific primer }\end{array}$ \\
\hline B. abortus S19 & IVRI* & 1 & 1154 \\
\hline B. abortus 544 & IVRI & 1 & 1154 \\
\hline B. melitensis $16 \mathrm{M}$ & IVRI & 1 & 745 \\
\hline B. ovis ATCC 25840 & NVSL** & 1 & 446 \\
\hline B. suis 1330 & IVRI & 1 & 290 \\
\hline B. suis ATCC 23445 & ATCC $* * *$ & 1 & 383 \\
\hline B. canis ATCC 23365 & NVSL & 1 & 224 \\
\hline Pseudomonas aeruginosa ATCC 27853 & ATCC & 1 & No amplification \\
\hline P. azotogenesis NCIM 2075 & NCIM $^{1}$ & 1 & No amplification \\
\hline Species and biovars & \multicolumn{3}{|l|}{ Host } \\
\hline B. abortus biovar 1 & Bovine & 16 & 1154 \\
\hline B. abortus biovar 2 & Bovine & 12 & 1154 \\
\hline B. abortus biovar 3 & Bovine & 9 & 1154 \\
\hline B. abortus biovar 4 & Bovine & 8 & 1154 \\
\hline B. abortus biovar 5 & Bovine & 2 & 1154 \\
\hline B. abortus biovar 6 & Bovine & 4 & 1154 \\
\hline B. abortus biovar 7 & Bovine & 2 & 1154 \\
\hline B. abortus biovar 9 & Bovine & 1 & 1154 \\
\hline B. melitensis biovar 1 & Human & 10 & 745 \\
\hline B. melitensis biovar 2 & Human & 6 & 745 \\
\hline B. melitensis biovar 3 & Human & 3 & 745 \\
\hline B. suis biovar 1 & Human & 2 & 290 \\
\hline B. suis biovar 2 & Human & 2 & 290 \\
\hline B. suis biovar 3 & Swine & 1 & 383 \\
\hline B. suis biovar 4 & Reindeer & 1 & 383 \\
\hline B. suis biovar 5 & Rodent & 1 & 383 \\
\hline Aeromonas hydrophila isolate DFRL1 & Human & 1 & No amplification \\
\hline Proteus mirabilis isolate DFRL1 & Human & 1 & No amplification \\
\hline Citrobacter freundii isolate DFRL1 & Human & 1 & No amplification \\
\hline Yersinia enterocolitica isolate DFRL1 & Human & 1 & No amplification \\
\hline
\end{tabular}

Table 1. Bacterial strains used in this study and the resultant PCR amplicon size (bp) with species-specific primer pair in monoplex PCR. *IVRI - Indian Veterinary Research Institute, Bareilly, Uttar Pradesh, India. **NVSL - National Veterinary Services Laboratories, Ames, Iowa. ***ATCC - American Type Culture Collection, USA. 'NCIM - National Collection of Industrial Microorganisms, Pune, India.

primer dimers. The strategy of designing primers is illustrated in Fig. 2. The primers were designed with the intent of obtaining specific yet easily distinguishable and interpretable amplicons under identical PCR conditions and were thus usable in the development of a multiplex PCR system. Designed primers were analyzed for specificity by using the BLASTN program prior to synthesis. To prevent false negative results due to the interference of inhibitory substances in test samples, competitive internal amplification control (IAC) was generated using the pUC19 plasmid as the target. The IAC primers were designed such that they had $5^{\prime}$ overhanging ends identical to the primer sequences of the B. abortus-specific primer pair and $3^{\prime}$ ends complementary to the pUC19 plasmid, amplifying $808 \mathrm{bp}$ of the pUC19 plasmid and yielding a product of $847 \mathrm{bp}$ (inclusive of the $5^{\prime} \mathrm{B}$. abortus-specific flanking regions of the primer pair). The sequences of the designed primers are presented in Table 2. All primers were synthesized by Eurofins India, India.

Monoplex PCR. For initial standardization of the detection system, monoplex PCR was performed using the primer sets and respective genomic DNA as template. Each $25 \mu \mathrm{L}$ PCR contained the following: $1 \mathrm{U}$ of Taq polymerase, $250 \mu \mathrm{M}$ each deoxynucleotide triphosphate, $1 \mathrm{x}$ PCR buffer (including $1.5 \mathrm{mM} \mathrm{MgCl} \mathrm{M}_{2}$ ), $10 \mathrm{pmol}$ of each oligonucleotide primer, and $25 \mathrm{ng}$ of template DNA. Thermal cycling was carried out in an Eppendorf thermal cycler (Eppendorf, USA) for 35 cycles of $30 \mathrm{~s}$ at $94^{\circ} \mathrm{C}$, annealing for $30 \mathrm{~s}$ at $56^{\circ} \mathrm{C}$, and extension at $72^{\circ} \mathrm{C}$ for $1 \mathrm{~min}$, with a final $8 \mathrm{~min}$ extension at $72^{\circ} \mathrm{C}$. Approximately $2 \mu \mathrm{l}$ of each PCR product was visualized by agarose gel electrophoresis. The template DNA of each species was used with its respective specific primer in each monoplex PCR.

IAC detection limit. The $847 \mathrm{bp}$ IAC amplicon amplified using chimeric oligonucleotide primers was purified using a QIAquick PCR Purification Kit (Qiagen, Germany) and further subjected to 1000 -fold serial dilutions from 1:1000 to 1:10000. From each dilution, $1 \mu \mathrm{l}$ of the amplicon was used as template along with $25 \mathrm{ng}$ of $B$. abortus gDNA and PCR-amplified in a single reaction using B. abortus-specific primers. The PCR components and amplification conditions were the same as described in the previous methods section. The lowest dilution of 
Whole genome sequence of 5 Brucella species $-B$. abortus,

$B$. melitensis, $B$. suis, $B$. ovis and $B$. canis from NCBI

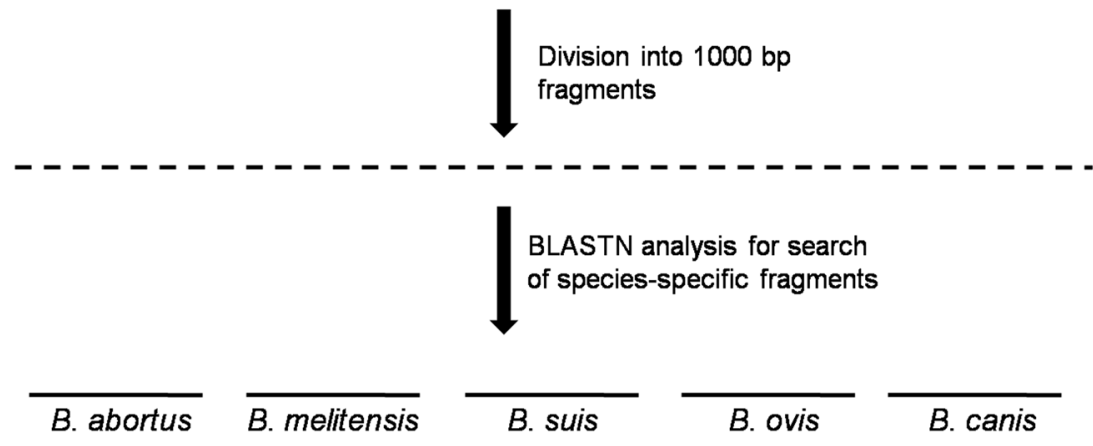

Figure 1. Scheme for mining Brucella species-specific nucleotide sequences.

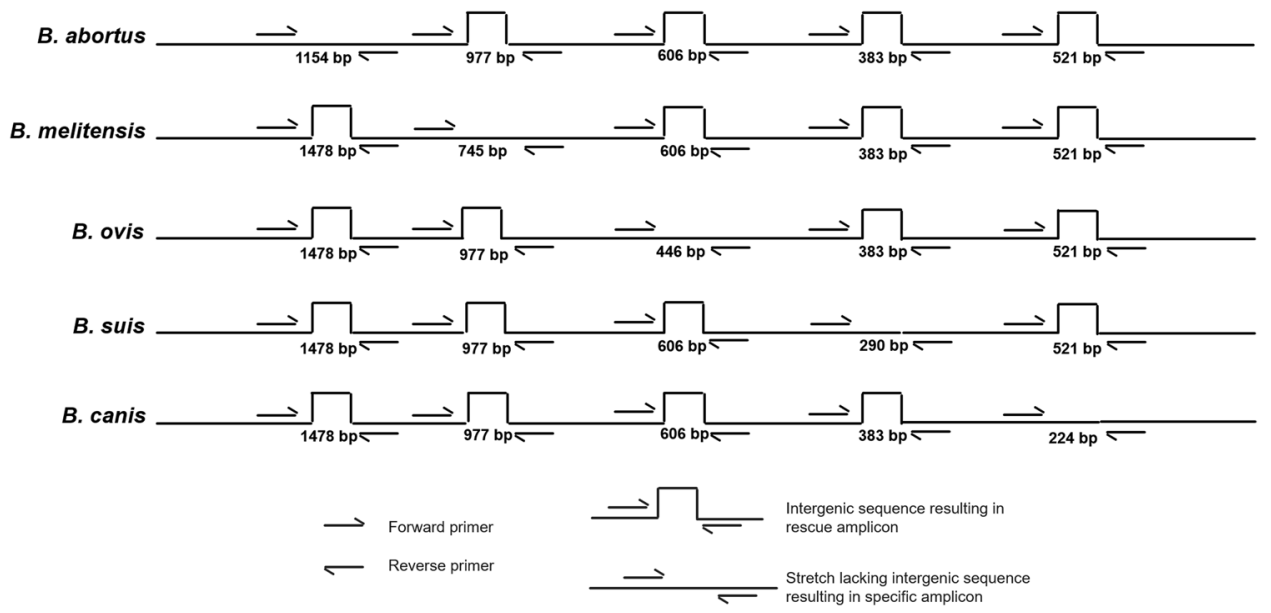

Figure 2. Pictorial illustration of the primer designing strategy using whole genome sequence for specific detection of B. abortus, B. melitensis, B. ovis, B. suis and B. canis.

IAC DNA resulting in an amplicon of the same intensity as B. abortus DNA was selected for standardization of multiplex PCR.

Multiplex PCR assay. Twenty-five nanograms of genomic DNA from each Brucella species, namely, B. abortus, B. melitensis, B. canis, B. suis and B. ovis, along with a 1:9000 dilution of IAC DNA, was subjected to each multiplex PCR in a $50 \mu \mathrm{l}$ reaction mixture containing $1 \mathrm{x}$ PCR buffer, $1.5 \mathrm{mM} \mathrm{MgCl}, 200 \mathrm{mM}$ each deoxynucleotide triphosphate, $10 \mathrm{pmol}$ of each oligonucleotide primer and $1 \mathrm{U}$ of Taq polymerase (Sigma Aldrich, India). PCR amplification was carried out as follows: initial denaturation at $94^{\circ} \mathrm{C}$ for $10 \mathrm{~min} ; 30 \mathrm{cycles}$ of $94^{\circ} \mathrm{C}$ for $1 \mathrm{~min}$, $56^{\circ} \mathrm{C}$ for $1 \mathrm{~min}$ and $72^{\circ} \mathrm{C}$ for $1.30 \mathrm{~min}$; and a final elongation step at $72^{\circ} \mathrm{C}$ for $8 \mathrm{~min}$. Amplicons were loaded onto a $2 \%$ agarose gel containing ethidium bromide that was run at $100 \mathrm{~V}$ for $1 \mathrm{~h}$. To evaluate the reproducibility of the developed assay, the PCR was repeated three times in three different thermal cyclers: Mastercycler Gradient (Eppendorf, USA), C1000 Touch ${ }^{\mathrm{TM}}$ (Biorad, USA) and Techne TC-3000G (Techne, UK).

Validation of the multiplex PCR assay for specificity. To evaluate the specificity of the multiplex PCR assay, purified genomic DNA isolated from all bacteria listed in Table 1 was employed. Nucleic acid was isolated using the QIAamp DNA Mini Kit (Qiagen) by following the manufacturer's instructions. Ten microliters of the eluted DNA was used to perform a multiplex PCR assay.

Statistical analysis. Descriptive statistics were performed to evaluate the specificity of the multiplex PCR assay. Reproducibility of the results for multiplex PCR optimization and overall specificity percentage was examined by repeating the blind experiments three times. Kappa statistical analysis was used to measure false negative results. Kappa estimates were defined as follows: values $<0.2$ imply poor agreement, values from $0.2-0.6$ imply fair to moderate, and values $>0.6$ imply good or very good agreement ${ }^{18}$. 


\begin{tabular}{|c|c|c|c|c|}
\hline \multirow[b]{2}{*}{ Primer ID } & \multirow[b]{2}{*}{ Sequence $\left(5^{\prime}\right.$ to $\left.3^{\prime}\right)$} & \multirow[b]{2}{*}{ DNA target } & \multicolumn{2}{|c|}{$\begin{array}{l}\text { Amplicon } \\
\text { size (bp) }\end{array}$} \\
\hline & & & Specific & Rescue \\
\hline BAA13334_I00002f & TGTATCGTACTTCATACTCC & \multirow{2}{*}{ Conserved hypothetical protein } & \multirow{2}{*}{1154} & \multirow{2}{*}{1478} \\
\hline BAA13334_I00002r & GCCGCAGAGACACGACAGAA & & & \\
\hline BMEA_A1673f & TCTCCTTGATTATGGTGCGA & \multirow{2}{*}{ Outer membrane protein, gene $O m p 31$} & \multirow{2}{*}{745} & \multirow{2}{*}{977} \\
\hline BMEA_A1673r & GACGTCTTGACCTTACCAT & & & \\
\hline BOV_1671f & GTTCCGGGCGACGCCAT & \multirow{2}{*}{ Phosphoglycerate kinase, gene $p g k$} & \multirow{2}{*}{446} & \multirow{2}{*}{606} \\
\hline BOV_1671r & GCAGCTTGGAGCATTATCC & & & \\
\hline BS1330_II0128f & AGTTGCCCCGCCTTCTGGAG & \multirow{2}{*}{$\begin{array}{l}\text { Flagellum-specific ATP synthase, gene } \\
\text { fliI }\end{array}$} & \multirow{2}{*}{290} & \multirow{2}{*}{383} \\
\hline BS1330_II0128r & TTCATAAATACGCGGCACAA & & & \\
\hline BCAN_A0740f & TTTTTCTGTCCACCATTTATC & \multirow{2}{*}{ GCN5-related N-acetyltransferase } & \multirow{2}{*}{224} & \multirow{2}{*}{521} \\
\hline BCAN_A0740r & ACAATAAAACAGTAGAGCCG & & & \\
\hline IAC-FP & TGTATCGTACTTCATACTCCAGCTGAATGAAGCCATACCA & \multirow{2}{*}{ pUC19 } & \multirow{2}{*}{\multicolumn{2}{|c|}{847}} \\
\hline IAC-RP & GCCGCAGAGACACGACAGAA $\underline{\text { AACCCGGTAAGACACGAC }}$ & & & \\
\hline
\end{tabular}

Table 2. List of primers used in multiplex PCR assay.

Data access. The respective specific and rescue nucleotide sequences of the B. abortus, B. melitensis, B. canis, $B$. suis and $B$. ovis reference strains used in this study were submitted to GenBank under the accession numbers MG888755 to MG888779 (Supplementary Table 2).

Ethical statement. This article does not contain any studies with human participants or animals performed by any of the authors.

\section{Results}

Mining of Brucella species-specific detection targets. On average, 3405 fragments were obtained after dividing the genomes of B. abortus A13334, B. melitensis ATCC 23457, B. ovis ATCC 25840, B. suis 1330, B. suis ATCC 23445, B. suis VBI22 and B. canis ATCC 23365 into $1000 \mathrm{bp}$ fragments. The derived fragments were analyzed by BLASTN analysis with genome sequences of Brucella strains available on the NCBI database, and a majority of the fragments showed high sequence identity (E values $<10^{-200}$ ). These conserved sequences from each species of Brucella were further aligned against the genomic sequences of the remaining four species to evaluate their uniqueness. This analysis revealed 25, 27, 37, 86 and 3 conserved nucleotide fragments in B. abortus, B. melitensis, B. suis, B. ovis and B. canis, respectively, and the fragments had a striking pattern of distribution among them. Specifically, a stretch of nucleotide sequence would be present in four species with $100 \%$ identity but completely absent in the fifth species. However, the flanking regions of that stretch of nucleotide sequence would be highly identical in all five species. For instance, a $1000 \mathrm{bp}$ fragment of $B$. abortus was present in B. melitensis, $B$. suis, B. ovis and B. canis with an additional $324 \mathrm{bp}$ unique interrupting intergenic nucleotide stretch within the fragment (that was strikingly absent in B. abortus), making the fragment $1324 \mathrm{bp}$ in length. Identical patterns were observed in the remaining four species of Brucella. Supplementary Figs. 1-5 (B. abortus, B. melitensis, B. suis, $B$. ovis and B. canis) represent multiple sequence alignments of randomly selected unique intergenic and/or intragenic sequences, one each from the five Brucella species of interest.

Brucella abortus specific fragment. The B. abortus-specific fragment of $1154 \mathrm{bp}$ and its respective rescue fragment of $1478 \mathrm{bp}$ from the four other Brucella species of interest were found to be comprised of an unknown conserved region $299 \mathrm{bp}$ from the forward primer position followed by a conserved hypothetical protein encoding region in an inverted position (Supplementary Fig. 6). Interestingly, no features of insertional sequences, including direct repeats, terminal inverted repeats, translational frame shifting or translational termination, were observed.

Brucella melitensis-specific fragment. The B. melitensis-specific fragment of $745 \mathrm{bp}$ was comprised of a C-terminal portion (including a stop codon) of an unknown gene encoding a hypothetical protein, an unannotated region $223 \mathrm{bp}$ length and a portion $(366 \mathrm{bp}$ ) of the gene encoding Omp31, including its open reading frame. In the case of its respective rescue fragment of $977 \mathrm{bp}$ from the other four Brucella species, an insertion of a truncated stretch of the ORF of a gene encoding the N-terminal portion of Omp31b within the ORF of the Omp31 gene was observed. Integration of this insertional sequence was accompanied by the duplication of a $14 \mathrm{bp}$ direct target repeat upstream of the insertional sequence (Supplementary Fig. 7).

Brucella suis-specific fragment. The B. suis-specific fragment of $290 \mathrm{bp}$ and its respective rescue fragment of $383 \mathrm{bp}$ from the four other Brucella species of interest were identified as a portion of the gene encoding flagellum-specific ATP synthase, fli, belonging to the flagellum-specific ATPase/type III secretory pathway virulence-related protein. ATPases of this group are responsible for the export of flagellum and virulence-related proteins. The bacterial flagellar motor is similar to the F0F1-ATPase, in that they both are proton-driven rotary molecular devices. However, the main function of the bacterial flagellar motor is to rotate the flagellar filament for cell motility. Intracellular pathogens such as Salmonella and Chlamydia also have proteins that are similar to 
the flagellar-specific ATPase but function in the secretion of virulence-related proteins via the type III secretory pathway. The gene was found to be directional within the genome of the Brucella species. Similar to the case of $B$. abortus-specific and rescue fragments, no features of insertional sequences, including direct repeats, terminal inverted repeats, translational frame shifting or translational termination, were observed (Supplementary Fig. 8).

Brucella canis-specific fragment. The B. canis-specific fragment of $224 \mathrm{bp}$ and its respective rescue fragment of $521 \mathrm{bp}$ from four other Brucella species of interest were identified to be the structural domain of the acyl-CoA acyltransferase enzyme from GNAT family $\mathrm{N}$-acetyltransferase. This domain has a 3-layer alpha/beta/ alpha structure that contains mixed beta-sheets and can be found in proteins including $\mathrm{N}$-acetyl transferase (NAT) family members, aminoglycoside $\mathrm{N}$-acetyltransferases, diamine acetyltransferase 1 , autoinducer synthetases such as protein LasI and acyl-homoserinelactone synthase EsaI, the histone acetyltransferase domain of P300/CBP-associating factor PCAF and the catalytic domain of GCN5 histone acetyltransferase. The insertional sequence had a length of $298 \mathrm{bp}$, and the structural domain of this gene encoding acyl-CoA acyltransferase was also annotated to be a part of this structural domain inserted between flanking direct "AT" repeats at the $128^{\text {th }}$ and $426^{\text {th }}$ nucleotide positions of the rescue fragment (Supplementary Fig. 9).

Brucella ovis-specific fragment. The B. ovis-specific fragment of $446 \mathrm{bp}$ comprised a C-terminal portion (336 bp) of phosphoglycerate kinase encoded by the $p g k$ gene, a $33 \mathrm{bp}$ DNA block encoding transposase, a $9 \mathrm{bp}$ direct repeat, a $35 \mathrm{bp}$ DNA block encoding DNA translocase and a $33 \mathrm{bp}$ DNA sequence encoding the N-terminus of a peptidase. On the other hand, the rescue fragment of the same conserved stretch in the other four Brucella species contained a 150 bp DNA block comprising an $87 \mathrm{bp}$ DNA fragment encoding an outer membrane autotransporter barrel domain, $24 \mathrm{bp}$ of an unknown nucleotide sequence and a $39 \mathrm{bp}$ truncated stretch of DNA sequence encoding a portion of a protein belonging to the $\mathrm{ABC}$ transporter ATP-binding protein group. The insertion of this $150 \mathrm{bp}$ DNA block was found to be after the direct repeat observed in the specific fragment (Supplementary Fig. 10).

PCR assay optimization. For initial standardization of the detection system, monoplex PCR was performed using each primer pair and respective genomic DNA as template. The result of each monoplex PCR is represented in Fig. 3. Monoplex PCR using B. abortus, B. melitensis, B. suis, B. ovis and B. canis in the presence of their specific primer pairs yielded amplicons of $1154 \mathrm{bp}, 745 \mathrm{bp}, 290 \mathrm{bp}, 446 \mathrm{bp}$ and $224 \mathrm{bp}$, respectively. Sequencing of each PCR amplicon revealed the complete identity of the amplified products with their specific genes. When the reaction was performed using genomic DNA from all five Brucella species in a single PCR test with any one pair of primers as mentioned in the methods section, each primer pair amplified a specific amplicon of a particular size with its respective species and a rescue amplicon with the remaining four species that was longer in length when compared to the respective specific amplicons; therefore, the amplicons were grouped into separate group altogether (Fig. 4). The rescue amplicons would provide additional information on the presence of other Brucella species in the test sample and hence rules out the chance of having nonspecific amplification with other contaminating organisms. For instance, the primer specific for B. abortus resulted in a 1154 bp amplicon with $B$. abortus and a $1478 \mathrm{bp}$ amplicon with four other species, namely, B. melitensis, B. suis, B. ovis and B. canis. The multiplex PCR described in this study includes a total of six pairs of primers: one pair for each of the five Brucella species, namely, B. abortus, B. melitensis, B. canis, B. suis and B. ovis, and one pair for the internal amplification control. In our multiplex PCR assay, each primer pair had the capability to detect a specific Brucella species and differentiate it from the other four species. This species-dependent differential amplification was noticed in the case of all specific primers, wherein the primer-specific species would result in an amplicon different from the amplicon size yielded by the remaining four species.

Interpretation of a multiplex PCR system. The multiplex PCR described here includes a total of six pairs of primers: one pair for amplification of each of the five Brucella species, namely, B. abortus, B. melitensis, B. canis, B. suis and B. ovis, and one for the internal amplification control (Fig. 5). Each pair of Brucella species primers mentioned above can yield PCR amplicons for all five species, resulting in a specific amplicon of a particular size with its respective species and a rescue amplicon longer than the specific amplicon with the other four species, thus differentiating them from the fifth Brucella species. For example, the B. abortus-specific primer pair results in a $1154 \mathrm{bp}$ amplicon with $B$. abortus and a $1478 \mathrm{bp}$ amplicon with 4 other species, namely, B. melitensis, B. suis, B. ovis and B. canis. The interpretation of the multiplex PCR assay described here is illustrated in Fig. 6. In an instance where multiplex PCR is performed with a test sample contaminated with all five Brucella species, namely, B. abortus, B. melitensis, B. suis, B. canis and B. ovis; the result will be as follows: (1) the B. abortus-specific primer pair yields an amplicon of $1154 \mathrm{bp}$ with $B$. abortus and $1478 \mathrm{bp}$ with the other four species; (2) the $B$. melitensis-specific primer pair yields an amplicon of $745 \mathrm{bp}$ with $B$. melitensis and an amplicon of $977 \mathrm{bp}$ with the other four species; (3) the B. suis-specific primer pair yields an amplicon of $290 \mathrm{bp}$ with B. suis and an amplicon of $383 \mathrm{bp}$ with the other four species; (4) the B. canis-specific primer pair yields an amplicon of $224 \mathrm{bp}$ with $B$. canis and an amplicon of $521 \mathrm{bp}$ with the other four species; (5) the B. ovis-specific primer pair yields an amplicon of $446 \mathrm{bp}$ with $B$. ovis and an amplicon of $606 \mathrm{bp}$ with the remaining four species; and (6) the IAC-specific primer yields an amplicon of $847 \mathrm{bp}$ with IAC DNA.

Assay specificity. The multiplex PCR assay developed was evaluated with 80 Brucella reference strains and isolates from humans and animals. The assay demonstrated a high rate of specificity (Table 1) in the case of $B$. abortus and B. melitensis irrespective of biovars with respect to their specific primers. All the biovars from these two species gave the same band profile, and the selected species were differentiated by the specific and rescue amplicon profiles. However, in the case of B. suis, biovars 3, 4 and 5 resulted in a 383 bp rescue amplicon with 


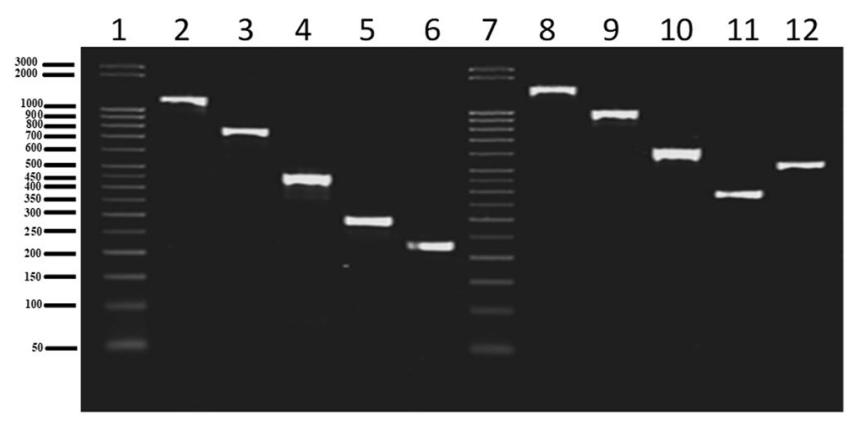

Figure 3. Agarose gel electrophoretic analysis of monoplex PCR format for specific detection of B. abortus, B. melitensis, B. ovis, B. suis and B. canis. Lane 1 and $7.50 \mathrm{bp}$ DNA ladder; Lane 2. B. abortus specific amplicon (1154 bp) with B. abortus specific primer; Lane 3. B. melitensis specific amplicon (745bp) with B. melitensis specific primer; Lane 4. B. ovis specific amplicon (446 bp) with B. ovis specific primer; Lane 5. B. suis specific amplicon (290 bp) with B. suis specific primer; Lane 6. B. canis specific amplicon (224bp) with B. canis specific primer; Lane 8 . B. abortus rescue amplicon $(1478 \mathrm{bp}$ ) with primers of the present study except $B$. abortus specific primer; Lane 9. B. melitensis rescue amplicon ( $977 \mathrm{bp}$ ) with primers of the present study except $B$. melitensis specific primer; Lane 10. B. ovis rescue amplicon $(606 \mathrm{bp})$ with primers of the present study except $B$. ovis specific primer; Lane 11 . B. suis rescue amplicon ( $383 \mathrm{bp}$ ) with primers of the present study except $B$. suis specific primer; Lane 12 . B. canis rescue amplicon $(521 \mathrm{bp})$ with primers of the present study except $B$. canis specific primer.

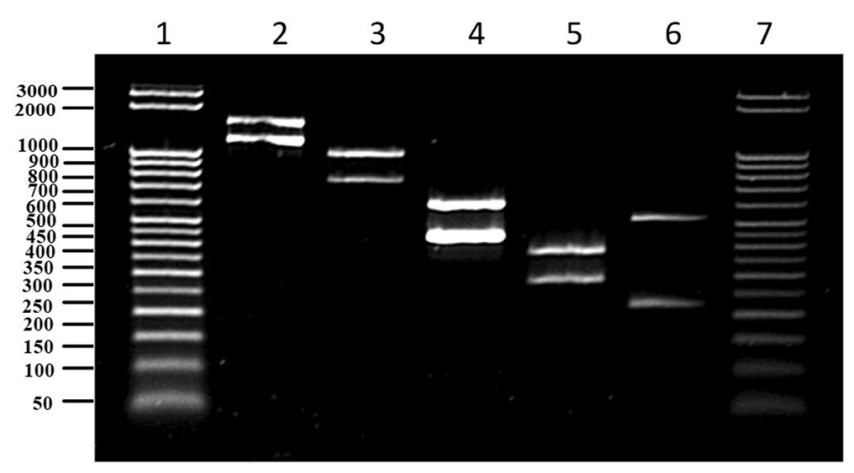

Figure 4. Agarose gel electrophoretic analysis of PCR assay using B. abortus, B. melitensis, B. ovis, B. suis and B. canis genomic DNA as template and one pair of primer per reaction. Lane 1 and $7.50 \mathrm{bp}$ DNA ladder; Lane 2. Specific and rescue amplicon resulted from B. abortus specific primer pair (1154bp and 1478 bp); Lane 3. Specific and rescue amplicon resulted from B. melitensis specific primer pair (745 bp and 977 bp); Lane 4. Specific and rescue amplicon resulted from B. ovis specific primer pair (446 bp and $606 \mathrm{bp}$ ); Lane 5. Specific and rescue amplicon resulted from $B$. suis specific primer pair (290 bp and $383 \mathrm{bp})$; Lane 6. Specific and rescue amplicon resulted from B. canis specific primer pair (224 bp and $521 \mathrm{bp}$ ).

B. suis-specific primers instead of the expected 290 bp amplicon, making the said biovars incompatible with the proposed strategy and indistinguishable from other species of Brucella. The B. suis ATCC 23445 strain also produced amplicons $383 \mathrm{bp}$ in length. The $B$. canis-specific primer pair resulted in a rescue amplicon $224 \mathrm{bp}$ in length with $B$. abortus biovar 5 instead of the expected $521 \mathrm{bp}$ amplicon. The $B$. ovis-specific primer pair resulted in a nonspecific amplicon $832 \mathrm{bp}$ in length with B. abortus biovar 5 instead of the expected $606 \mathrm{bp}$ amplicon. These exceptional cases were encountered even during the in silico analysis of the designed primers for reliability in multiplex PCR. No amplification was recorded with the 6 non-Brucella species tested.

\section{Discussion}

The characteristic feature of bacterial ISs is their capacity to generate mutations in an organism by transposition within the genome. ISs occur in various copy numbers in a particular genome and can move within the genome or even between genomes horizontally, resulting in clonal expansion of species in a genus ${ }^{19}$. Intensive studies with respect to insertion sequences have unraveled genetic features, namely, direct repeats, terminal inverted repeats, translational frame shifting or translational termination, segmental genome duplication and several other features associated with genetic insertion and/or translocation of DNA blocks in bacterial genomes ${ }^{20}$.

The transposition of ISs is generally identified by the presence of short terminal inverted repeats that flank these DNA blocks. Many ISs also carry one or two open reading frames encoding genes that are essential for their transposition. The mechanism of transposition is primarily dependent on catalysts, including transposase and inverted repeats necessary for the binding of transposase, which in turn results in donor DNA cleavage and strand 


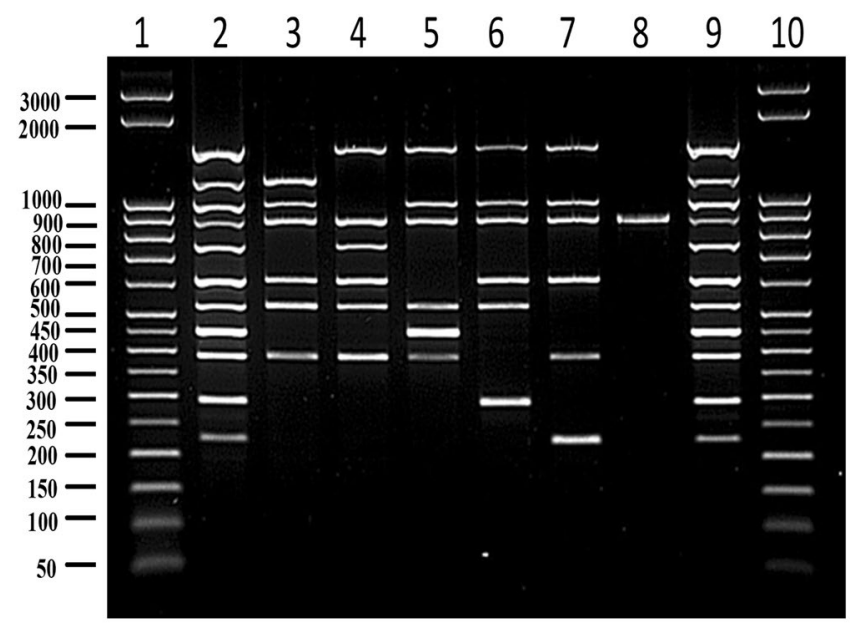

Figure 5. Agarose gel electrophoretic analysis of multiplex PCR assay optimized by incorporation of an internal amplification control $(847 \mathrm{bp})$. All five novel primer pairs were involved in the PCR reaction. Lane 1 and 10. 50 bp DNA ladder; Lane 2 and 9. PCR using B. abortus, B. melitensis, B. ovis, B. suis and B. canis genomic DNA as template DNA; Lane 3. PCR using B. abortus genomic DNA as template DNA; Lane 4. PCR using $B$. melitensis genomic DNA as template DNA; Lane 5. PCR using B. ovis genomic DNA as template DNA; Lane 6. PCR using $B$. suis genomic DNA as template DNA; Lane 7. PCR using B. canis genomic DNA as template DNA; Lane 8. PCR without any genomic DNA.

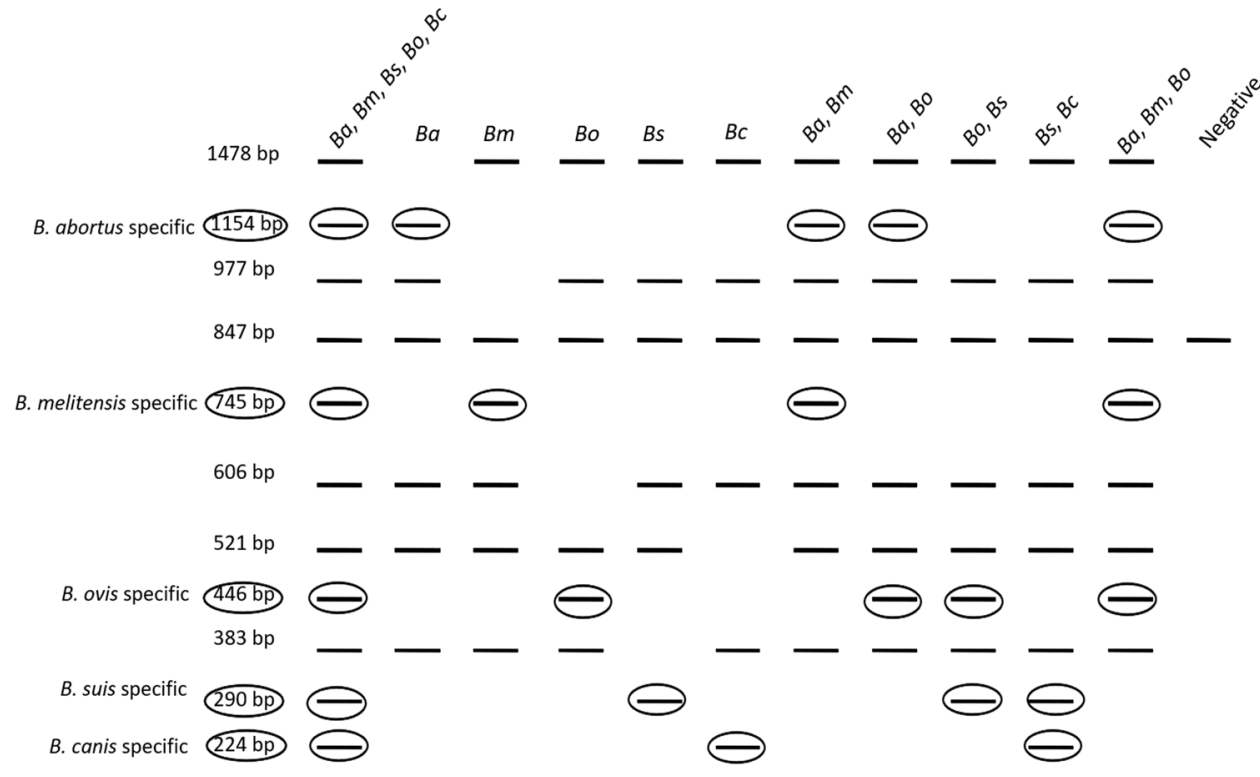

Figure 6. Figure illustrating the interpretation of multiplex PCR formats for specific detection of Brucella species. Lane1: Specific PCR amplicons of all five Brucella species are observed for species identification and differentiation; Lane 2: Specific PCR amplicon of 1154 bp indicates presence of B. abortus; Lane 3: Specific PCR amplicon of $745 \mathrm{bp}$ indicates presence of $B$. melitensis; Lane 4: Specific PCR amplicon of 446 bp indicates presence of B. ovis; Lane 5: Specific PCR amplicon of $290 \mathrm{bp}$ indicates presence of B. suis; Lane 6: Specific PCR amplicon of $224 \mathrm{bp}$ indicates presence of B. canis; Lane 7: Specific PCR amplicons of $1154 \mathrm{bp}$ and $745 \mathrm{bp}$ indicates presence of B. abortus and B. melitensis; Lane 8: Specific PCR amplicons of $1154 \mathrm{bp}$ and $446 \mathrm{bp}$ indicates presence of B. abortus and B. ovis; Lane 9: Specific PCR amplicons of $446 \mathrm{bp}$ and $290 \mathrm{bp}$ indicates presence of B. ovis and B. suis; Lane 10: Specific PCR amplicons of $290 \mathrm{bp}$ and $224 \mathrm{bp}$ indicates presence of B. suis and B. canis; Lane 11: Specific PCR amplicons of $1154 \mathrm{bp}, 745 \mathrm{bp}$ and $446 \mathrm{bp}$ indicates presence of $B$. abortus, B. melitensis and B. ovis; Lane 12: PCR amplicon of $847 \mathrm{bp}$ only indicates that the sample is negative for contamination with any of the five Brucella species.

transfer ${ }^{20}$. The presence and transposition of IS elements within and between genomes are associated with gene inactivation, modulating virulence, the expression of neighboring genes, antibiotic resistance and metabolism ${ }^{21}$. Irrespective of IS families, elevated transposition of ISs between genomes serves to amplify both deleterious 
and advantageous fitness changes, but this accelerates the extinction of elements, particularly if the mean fitness effect of IS-induced mutations is negative. However, transposition bursts create genetic diversity, which occasionally generates advantageous mutations and thus help organisms adapt to new environments ${ }^{22}$. In this line of IS-mediated mutations among prokaryotes, several bacterial models, including Enterobacteriaceae members, Pseudomonas sp., Acinetobacter baumannii, Lactobacillus sp., Burkholderia sp., Staphylococcus aureus and Vibrio sp. have been successfully explored and studied. These studies have supported the establishment of a knowledge base on the involvement of ISs in the plasticity and adaptability of bacterial genomes ${ }^{21}$. However, in the case of the genus Brucella, very little information is available on the diversity of ISs within the genomes, and only IS711 has been extensively studied in this respect until now. With this background, we designed our study to explore novel genetic polymorphisms among Brucella species that can be exploited to establish the differentiation among them for diagnostic applications.

The distinctively remarkable pattern of insertions and deletions of insertional sequences with precise lengths with the determined target sequences in our study, which was exploited in the development of a novel multiplex PCR assay for use in the diagnosis of brucellosis, encouraged us to understand the mechanism involved in the presence or absence of such precise lengths of IS elements. Our opinion was that one mechanism was possibly involved in the unique pattern of inserting DNA blocks with identical lengths in four different species of Brucella, but not one species. To our surprise, the diversity was not limited to the range of complexity observed among each fragment but to the possible mechanisms involved in the transposition of the DNA blocks.

The typical segmental genome duplication observed in the case of the insertion of the Omp31b gene fragment within the conserved $O m p 31$ gene is a feature usually observed among the IS 21 family. Segmental duplications usually occur at more than one site within a given genome with a high level of sequence identity, as confirmed by both in silico and in situ hybridization experiments, and such patterns are usually observed in the human genome ${ }^{23,24}$. Such segmental duplications are observed as an indicative sign of evolutionary rearrangement ${ }^{25,26}$.

In the case of the B. abortus and B. suis rescue fragments in our study, no features of insertional sequences were observed. A lack of such features is an indication of possible homologous intermolecular or intramolecular recombination that would have occurred between two insertional elements each with different direct repeats. Such recombination usually results in the formation of a hybrid element carrying one direct repeat of each parent. In some cases, formation of adjacent deletions from duplicative intramolecular transpositions can also result in such insertional sequence elements. A single copy of the direct repeat could be located on each of the deletion products in such cases. However, extensive studies are required to confirm the exact mechanism involved in such insertion events on a case-by-case basis throughout the genome.

The generation of short directly repeated sequences flanking the inserted DNA sequences is a general feature of insertional sequence elements. Such insertions are facilitated by the attack of each DNA strand at the target direct repeat site by one of the two transposon ends in a staggered way. Usually, the lengths of such direct repeats range between 2 and $14 \mathrm{bp}$. In the case of the $B$. canis rescue fragment, the flanking direct repeat was $2 \mathrm{bp}$ in length. Usually, in the case of such direct repeats, the insertion sequence will be generated as a genome duplication of a fixed length, but nevertheless, generation of small variations in the transposition complex is also observed at a low frequency. Latter condition was observed in the rescue fragment of $B$. canis in our study.

The intention of the present work was to develop a detection system for simultaneous and differential identification of five Brucella species irrespective of biovars and strains based on comparative genomic analysis using the updated database of Brucella genomes. The specificity of the intended detection system is crucial for the diagnosis of brucellosis, which solely depends on the target genes. With the comparative whole genomic analysis approach for mining species-specific nucleotide sequences, a remarkable pattern of insertion or deletion of certain length nucleotides was encountered in the genomes of B. abortus, B. melitensis, B. suis, B. ovis and B. canis. Interestingly, the deciphered sequences were not limited to genes required for virulence or other specialized metabolic functions but also included noncoding sequences.

The distinctively remarkable pattern of insertions and deletions of nucleotide sequences or DNA blocks with particular lengths with the determined target sequences separated them into two groups, one group with deletions and the other four groups with insertions, supporting the development of the exemplary detection tool. The unique sequences were differentially present in four species but absent in one and were intergenic and/or intragenic in nature, inserting within conserved regions of the Brucella genus as detection targets. Species-specific deletions in the case of Brucella were reported earlier by Rajashekara and coworkers (2004) based on microarray analysis, and these deletions were found to be highly conserved. Successful exploitation of those deletions in the design of species-specific primers for the differentiation of $B$. neotomae and B. canis was described in the previously mentioned study. The primers in that study not only resulted in different amplicon sizes in the cases of B. neotomae and B. canis but also showed one or completely different PCR amplification bands in the case of the other Brucella species screened. Similar differential patterns were derived for specific and differential identification of other Brucella species by the exploitation of different deletions. However, as a limitation in that study, biovars within a species showed different banding patterns, making interpretation a difficult and challenging task ${ }^{27}$.

In the current study, irrespective of biovars, in the unique sequence of $B$. abortus, which we selected randomly, to include as a detection target, a deletion of $324 \mathrm{bp}$ was observed between positions 469 and 470 when base count was performed from the hybridizing position of the B. abortus forward primer, resulting in a $1154 \mathrm{bp}$ specific amplicon and a $1478 \mathrm{bp}$ rescue amplicons in the other four species. Similarly, upon referring to the forward primer hybridizing position as the base count in the case of the other four species, the $745 \mathrm{bp}$ specific amplicon of $B$. melitensis had a deletion of $232 \mathrm{bp}$ between positions 422 and 423 compared to the $977 \mathrm{bp}$ rescue amplicons of the other four species. The B. ovis-specific amplicon (446 bp) had a deletion of $160 \mathrm{bp}$ between the $379^{\text {th }}$ and $380^{\text {th }}$ positions, differentiating it from the $606 \mathrm{bp}$ rescue amplicon. A $93 \mathrm{bp}$ nucleotide stretch was deleted from the $290 \mathrm{bp}$ specific amplicon of $B$. suis between its $109^{\text {th }}$ and $110^{\text {th }}$ nucleotide positions. When compared with 
the rescue amplicons of B. canis, its specific amplicon had a $297 \mathrm{bp}$ deletion from the $129^{\text {th }}$ nucleotide position, making it a 224 bp product.

Five pairs of oligonucleotide primers were designed to exploit the newly identified species-specific nucleotide sequences for the development of a multiplex PCR assay that identifies five Brucella species in one single reaction. Initially, each species-specific primer was exploited in the development of a monoplex PCR system for each species with specific amplicons of $1154 \mathrm{bp}, 745 \mathrm{bp}, 446 \mathrm{bp}, 290 \mathrm{bp}$ and $224 \mathrm{bp}$ for B. abortus, B. melitensis, B. ovis, $B$. suis and $B$. canis, respectively. However, when the same five species-specific primer pairs were employed in a multiplex PCR for the detection of each species, a unique multiplex PCR system resulted. This was because all the primers would anneal to the conserved regions flanking all five nucleotide sequences of the Brucella genome, resulting in four additional amplicons termed "rescue amplicons" along with the species-specific amplicon. Nevertheless, in a multiplex PCR in the presence of all five Brucella species, an integrative format comprising all the species-specific amplicons was obtained for the identification and differentiation of all five species together in a single lane by gel electrophoresis. Furthermore, these primers were employed in the stabilization of conventional monoplex and multiplex PCR systems. To avoid false negative results, competitive internal amplification control (IAC) was also incorporated in the multiplex PCR system with pUC19 plasmid DNA.

The stabilized monoplex and multiplex PCRs were evaluated for specificity with reference strains of the said five Brucella species as well as non-Brucella species. As an outcome, irrespective of biovars, a highly specific PCR-based detection format was developed that could successfully and differentially identify $B$. abortus, $B$. melitensis, B. ovis, B. suis and B. canis. The major advantage of our assay is that the use of any one primer pair was also sufficient to identify Brucella at the genus level and species level by seeking specific and/or rescue amplicons irrespective of biovars. Each pair of primers was useful not only as a species-specific marker but also as a genus-specific marker.

The developed multiplex PCR was used to evaluate its applicability in detecting 80 strains of Brucella species. The assay successfully identified the five target species and differentiated them from each other. This result reflected the unambiguous specificity of the assay and thus its applicability in the diagnosis of brucellosis. However, to examine the robustness of this tool in clinical cases, multiplex PCR assays require extensive validation in several reference laboratories with a large number of clinical samples.

Received: 23 September 2019; Accepted: 2 March 2020;

Published online: 14 April 2020

\section{References}

1. El-Sayed, A. \& Awad, W. Brucellosis: Evolution and expected comeback. Int. J. Vet. Sci. Med. 6, S31-S35 (2018).

2. Scholz, H. \& Vergnaud, G. Molecular characterisation of Brucella species. Rev. Sci. Tech. OIE 32, 149-162 (2013).

3. Garcia-Yoldi, D. Multiplex PCR assay for the identification and differentiation of all Brucella species and the vaccine strains Brucella abortus S19 and RB51 and Brucella melitensis Rev1. Clin. Chem. 52, 779-781 (2006).

4. Marianelli, C. et al. Use of MLVA-16 typing to trace the source of a laboratory-acquired Brucella infection. J. Hosp. Infect. 68, 274-276 (2008).

5. Kattar, M. M. et al. Evaluation of a multilocus variable-number tandem-repeat analysis scheme for typing human Brucella isolates in a region of brucellosis endemicity. J. Clin. Microbiol. 46, 3935-3940 (2008).

6. Huber, B., Scholz, H. C., Lucero, N. \& Busse, H. J. Development of a PCR assay for typing and subtyping of Brucella species. Int. J. Med. Microbiol. 299, 563-573 (2009).

7. Valdezate, S., Navarro, A., Villalon, P., Carrasco, G. \& Saez-Nieto, J. A. Epidemiological and phylogenetic analysis of Spanish human Brucella melitensis strains by multiple-locus variable-number tandem-repeat typing, hypervariable octameric oligonucleotide fingerprinting, and rpoB typing. J. Clin. Microbiol. 48, 2734-2740 (2010).

8. Lopez-Goni, I. et al. Evaluation of a multiplex PCR assay (Bruce-ladder) for molecular typing of all Brucella species, including the vaccine strains. J. Clin. Microbiol. 46, 3484-3487 (2008).

9. Ferreira, A. C. et al. MLVA16 typing of Portuguese human and animal Brucella melitensis and Brucella abortus isolates. PLoS ONE. 7, e42514 (2012).

10. Ocampo-Sosa, A. A., Agüero-Balbín, J. \& García-Lobo, J. M. Development of a new PCR assay to identify Brucella abortus biovars 5, 6 and 9 and the new subgroup 3 b of biovar 3. Vet. Microbiol. 110, 41-51 (2005).

11. Halling, S. M. et al. Completion of the genome sequence of Brucella abortus and comparison to the highly similar genomes of Brucella melitensis and Brucella suis. J. Bacteriol. 187, 2715-2726 (2005).

12. Bricker, B. J., Ewalt, D. R., MacMillan, A. P., Foster, G. \& Brew, S. Molecular characterization of Brucella strains isolated from marine mammals. J. Clin. Microbiol. 38, 1258-1262 (2005).

13. Zygmunt, M. S., Maquart, M., Bernardet, N., Doublet, B. \& Cloeckaert, A. Novel IS711-specific chromosomal locations useful for identification and classification of marine mammal Brucella strains. J. Clin. Microbiol. 48, 3765-3769 (2010).

14. Tsolis, R. M. et al. Genome degradation in Brucella ovis corresponds with narrowing of its host range and tissue tropism. PLoS ONE. 4, e5519 (2009).

15. Mancilla, M., Ulloa, M., López-Goñi, I., Moriyón, I. \& María Zárraga, A. Identification of new IS711 insertion sites in Brucella abortus field isolates. BMC Microbiol. 11, 176 (2011).

16. Bricker, B. J. \& Halling, S. M. Differentiation of Brucella abortus bv. 1, 2, and 4,Brucella melitensis, Brucella ovis, and Brucella suis bv. 1 by PCR. J. Clin. Microbiol. 32, 2660-2666 (1994).

17. Gyuranecz, M. et al. Natural IS711 insertion causing omp31 gene inactivation in. Brucella ovis. J. Vet. Diagn. Invest. 25, 234-238 (2013).

18. Altman, D. G. Statistics in medical journals: Developments in the 1980s. Statist. Med. 10, 1897-1913 (1991).

19. Robinson, D. G., Lee, M.-C. \& Marx, C. J. OASIS: an automated program for global investigation of bacterial and archaeal insertion sequences. Nucleic Acids Res. 40(22), e174-e174 (2012).

20. Mahillon, J. \& Chandler, M. Insertion Sequences. Microbiol. Mol. Biol. Rev. 62(3), 725-774 (1998).

21. Vandecraen, J., Chandler, M., Aertsen, A. \& Van Houdt, R. The impact of insertion sequences on bacterial genome plasticity and adaptability. Crit. Rev. Microbiol. 43, 709-730 (2017).

22. Wu, Y., Aandahl, R. Z. \& Tanaka, M. M. Dynamics of bacterial insertion sequences: can transposition bursts help the elements persist? BMC Evol. Biol. 15, 288 (2015).

23. Cheung, V. G. et al. Integration of cytogenetic landmarks into the draft sequence of the human genome. Nature. 409(6822), 953 (2001). 
24. She, X. et al. The structure and evolution of centromeric transition regions within the human genome. Nature. 430, 857-864 (2004).

25. Ji, Y. Structure of Chromosomal Duplicons and their Role in Mediating Human Genomic Disorders. Genome Res. 10, 597-610 (2000).

26. Bailey, J. A., Baertsch, R., Kent, W., Haussler, D. \& Eichler, E. E. Hotspots of mammalian chromosomal evolution. Genome Biol. 5, R23 (2004).

27. Rajashekara, G., Glasner, J. D., Glover, D. A. \& Splitter, G. A. Comparative whole-genome hybridization reveals genomic islands in Brucella species. J. Bacteriol. 186, 5040-5051 (2004).

\section{Acknowledgements}

The first author is thankful to Indian Council of Medical Research (ICMR), India for support. Second author is thankful to Department of Science and Technology (DST), India for support through INSPIRE program. This research did not receive any specific grant from funding agencies in the public, commercial, or not-for-profit sectors.

\section{Author contributions}

S.P. and H.V.B. made the study design and S.P., P.V.B. and M.G. performed the experiments and S.N. prepared figures and S.P. and P.V.B. prepared the manuscript and S.R., B.K. and H.V.B. reviewed and approved the manuscript.

\section{Competing interests}

The authors declare no competing interests.

\section{Additional information}

Supplementary information is available for this paper at https://doi.org/10.1038/s41598-020-62472-3.

Correspondence and requests for materials should be addressed to H.V.B.

Reprints and permissions information is available at www.nature.com/reprints.

Publisher's note Springer Nature remains neutral with regard to jurisdictional claims in published maps and institutional affiliations.

(c) Open Access This article is licensed under a Creative Commons Attribution 4.0 International License, which permits use, sharing, adaptation, distribution and reproduction in any medium or format, as long as you give appropriate credit to the original author(s) and the source, provide a link to the Creative Commons license, and indicate if changes were made. The images or other third party material in this article are included in the article's Creative Commons license, unless indicated otherwise in a credit line to the material. If material is not included in the article's Creative Commons license and your intended use is not permitted by statutory regulation or exceeds the permitted use, you will need to obtain permission directly from the copyright holder. To view a copy of this license, visit http://creativecommons.org/licenses/by/4.0/.

(c) The Author(s) 2020 